\title{
Generalizing Ham Sandwich Cuts to Equitable Subdivisions*
}

\author{
S. Bespamyatnikh, D. Kirkpatrick, and J. Snoeyink \\ Department of Computer Science, University of British Columbia, \\ Vancouver, British Columbia, Canada V6T 1 Z4 \\ \{besp,kirk,snoeyink\}@cs.ubc.ca
}

\begin{abstract}
We prove a generalization of the famous Ham Sandwich Theorem for the plane. Given $g n$ red points and $g m$ blue points in the plane in general position, there exists an equitable subdivision of the plane into $g$ disjoint convex polygons, each of which contains $n$ red points and $m$ blue points. For $g=2$ this problem is equivalent to the Ham Sandwich Theorem in the plane. We also present an efficient algorithm for constructing an equitable subdivision.
\end{abstract}

\section{Introduction}

The planar case of the well-known discrete Ham Sandwich Theorem [18] states that, for finite sets of red and blue points in the plane, there exists a line dividing both red and blue points into sets of equal size. The Ham Sandwich problem is well studied from an algorithmic point of view [2], [5]-[7], [12]-[14], [17], [19]. An optimal algorithm of Lo et al. [13] finds a Ham Sandwich cut in linear time. Kaneko and Kano [11] considered balanced partitions of two sets in the plane. They gave the following conjecture.

Conjecture 1. Let $m \geq 2, n \geq 2$, and $g$ be positive integers. Let $R$ and $B$ be two disjoint sets of points in the plane such that no three points of $R \cup B$ are collinear, $|R|=g n$ and $|B|=g m$. Then $R \cup B$ can be partitioned into $g$ subsets $P_{1}, \ldots, P_{g}$ satisfying the following two conditions: (i) $P_{i}$ and $P_{j}$ are linearly separable for all $1 \leq i<j \leq g$; and (ii) $\left|P_{i} \cap R\right|=n$ and $\left|P_{i} \cap B\right|=m$ for all $1 \leq i \leq g$.

For $g=2$ the conjecture is equivalent to the Ham Sandwich Theorem [18, p. 212]. Kaneko and Kano proved the conjecture in the case of $n \leq 2$. In this paper we prove their

\footnotetext{
* Support from NSERC is gratefully acknowledged.
} 


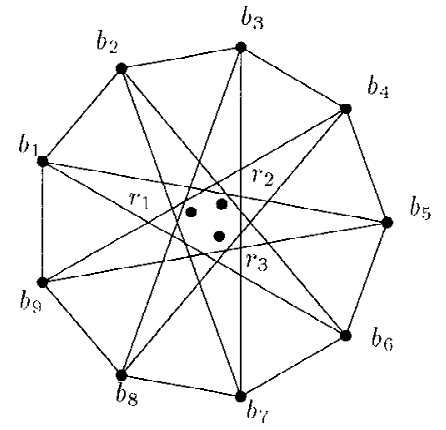

(a)

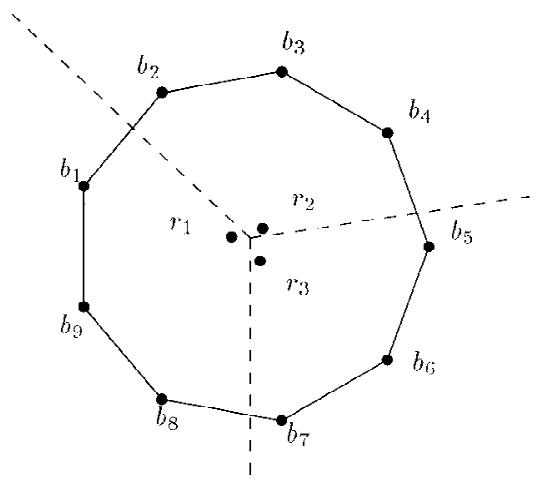

(b)

Fig. 1. An example of three red points $r_{1}, r_{2}$, and $r_{3}$ and nine blue points $b_{1}, \ldots, b_{9}$ for $g=3$. (a) There is no equitable 2-cutting. (b) An equitable 3-cutting.

conjecture. Actually we prove a stronger theorem providing what we call an equitable subdivision of the plane.

Note that, for $g=2^{k}$, Conjecture 1 can be proved by applying the Ham Sandwich Theorem in a divide-and-conquer fashion. For general $g$, such a strategy is not possible. Specifically, Fig. 1 illustrates an example of three red and nine blue points that does not admit an equitable 2-cutting, that is, a partition of the plane by a line such that the $i$ th halfplane contains $g_{i} n$ red and $g_{i} m$ blue points for some integers $g_{1}, g_{2}<g$ (in other words, there is no halfplane with exactly one red and three blue points). However, it is sufficient to apply an equitable 3-cutting. An equitable 3-cutting is a partition of the plane by three rays with a common apex into three convex wedges, each of which contains a proportional number of red and blue points. In other words, the $i$ th wedge contains $g_{i} n$ red points and $g_{i} m$ blue points, for some $0 \leq g_{1}, g_{2}, g_{3}<g$ with $g_{1}+g_{2}+g_{3}=g$.

Theorem 2 (3-Cutting). For any gn red points $(g \geq 2)$ and gm blue points in the plane in general position, there is equitable 3-cutting of red and blue points.

When $g=2$, Theorem 2 is equivalent to the Ham Sandwich Theorem. For $g \geq 3$, we can apply Theorem 2 recursively for the wedges with $g_{i} \geq 2$. This produces what we refer to as an equitable subdivision of the plane. We present an algorithm ${ }^{1}$ for producing such an equitable subdivision in $O\left(N^{4 / 3} \log ^{3} N \log g\right)$ time where $N=g(n+m)$ is the total number of points. Note we need to use the fact that an equitable 3 -cutting defines a convex partition in order to preserve connectivity of the regions produced in the recursive application of Theorem 2 .

\footnotetext{
${ }^{1}$ A Java demo is available at http://www.cs.ubc.ca/spider/besp/ham.htm.
} 
Very recently the conjecture of Kaneko and Kano has been independently proven by Ito et al. [10] and Sakai [16]. Comparing our result with papers [10] and [16] we can say that they prove essentially the same 3-cutting theorems but both use different (and somewhat more complicated) techniques. Ito et al. [10] prove the existence of a 3-cutting with one ray passing through a fixed point on the convex hull of the union of the red and blue points. Sakai [16] gives a direct proof of the continuous version of the 3-Cutting Theorem using a partition of the plane into square cells of an appropriate size. Paper [10] refers only to the discrete version. Neither of the papers present efficient algorithms.

Bárány and Matoušek [3] consider partitions of the plane, $k$-fans, using $k$ lines and specified weights of $k$ regions. They prove a weaker version of Theorem 2, namely the existence of a 3-fan that defines a not necessarily convex subdivision.

\section{Preliminaries}

Let $R$ be a set of $g n$ red points and let $B$ be a set of $g m$ blue points in the plane in general position, i.e., there are no three points lying on the same line. The integer $g$ is the number of groups into which the sets $R$ and $B$ are to be divided. A 2-cutting is a partition of the plane by the line into two halfplanes. A 2-cutting is equitable (or more specifically $\left(g_{1}, g_{2}\right)$-equitable) if the $i$ th open halfplane contains exactly $g_{i} n$ red and $g_{i} m$ blue points for some integers $g_{1}, g_{2}<g$ with $g_{1}+g_{2}=g$.

If there is a $\left(g_{1}, g_{2}\right)$-equitable 2-cutting of the red and blue points, then the problem can be reduced to the subproblems for the number of groups $g_{1}$ and $g_{2}$. Otherwise, for any $g_{1} \in\{1, \ldots, g-1\}$, a halfplane containing exactly $g_{1} n$ red points (we call it a $g_{1}$-halfplane) contains either less than or greater than $g_{1} m$ blue points. We assign to the halfplane the sign -1 or +1 , respectively.

Lemma 3. If, for some $g_{1} \in\{1, \ldots, g-1\}$, there are two $g_{1}$-halfplanes with opposite signs, then there is a $\left(g_{1}, g_{2}\right)$-equitable 2-cutting.

Proof. Lemma 3 is the same as Lemma 2 of [11]. We prove the result using geometric duality exploiting an interpretation in terms of $k$-levels of the dual arrangement associated with the point set $R \cup B$. The dual of a point $p=\left(p_{1}, p_{2}\right)$ is the line $y=-p_{1} x+p_{2}$, and the dual of a line $y=a x+b$ is the point $(a, b)$.

Let $l_{1}$ and $l_{2}$ be the lines that define two $g_{1}$-halfplanes with signs -1 and +1 . We can rotate the plane so that the lines become nonvertical and the $g_{1}$-halfplanes become lower halfplanes. In the dual setting the red and blue points correspond to red and blue lines. The $k$-level of an arrangement of lines is a polygonal line formed by all edges of level $k$, where the level of an edge is the number of lines lying strictly below its interior point. The point dual of an equitable 2-cutting lies between the $g_{1} n$-level and $\left(g_{1} n+1\right)$-level of red lines and between the $g_{1} m$-level and $\left(g_{1} m+1\right)$-level of blue lines. An equitable 2-cutting can be found if two levels of different colors cross, see Fig. 2. Otherwise the entire $\left(g_{1} n+1\right)$-level of red lines lies below the $g_{1} m$-level of blue lines or the $g_{1} n$-level of red lines lies above the $\left(g_{1} m+1\right)$-level of blue lines. In this case the points $p_{1}, p_{2}$ dual to $l_{1}, l_{2}$ lie below the $g_{1} m$-level of blue lines or above the $\left(g_{1} m+1\right)$-level of blue 


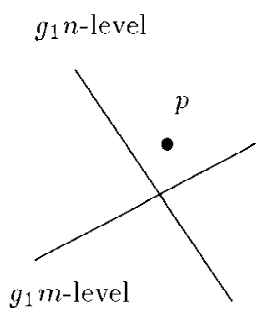

(a)

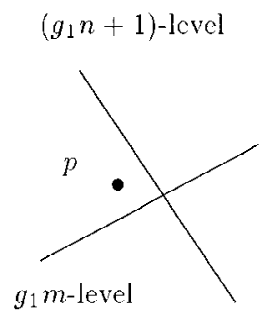

(b)

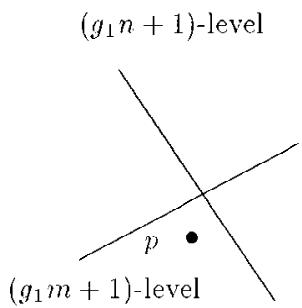

(c)

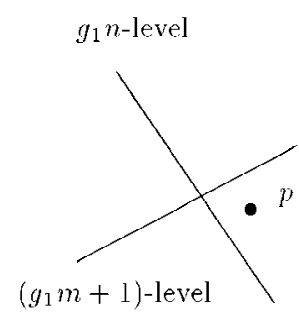

(d)

Fig. 2. The point $p$ is the dual of an equitable 2-cutting.

lines (the points $p_{1}, p_{2}$ lie between the $g_{1} n$-level and $\left(g_{1} n+1\right)$-level of red lines). It is impossible because the point $p_{1}$ is below the $g_{1} m$-level and the point $p_{2}$ is above the $\left(g_{1} m+1\right)$-level of blue lines.

Lemma 3 allows us to assume that, for any $g_{1}$, all $g_{1}$-halfplanes have the same sign. However, in our algorithm we avoid the computation of all signs. Instead we use Lemma 3 to find an equitable 2-cutting if in the course of the algorithm it is discovered that two $g_{1}$-halfplanes have opposite signs for some $g_{1}$. To begin we define signs for a canonical set of halfplanes. Draw $g-1$ vertical lines that divide red points into $g$ sets of $n$ points. We assume that there are no two points lying on the same vertical line (otherwise we can rotate the points). We define the sign of $g_{1}, s\left(g_{1}\right)$, as the sign of the left halfplane formed by the $g_{1}$ th vertical line.

A 3-cutting is a partition of the plane into three wedges $W_{1}, W_{2}$, and $W_{3}$ by three rays with a common point that is called the apex of the 3-cutting. A 3-cutting is convex if its wedges are convex. A convex 3-cutting is equitable (or more specifically $\left(g_{1}, g_{2}, g_{3}\right)$ equitable) if the open wedge $W_{i}$ contains exactly $g_{i} n$ red and $g_{i} m$ blue points for some integers $0 \leq g_{1}, g_{2}, g_{3}<g$ with $g_{1}+g_{2}+g_{3}=g$. Note that an equitable 2-cutting is an equitable 3-cutting for some $g_{i}=0$.

We will find a 3-cutting with one ray going down, see Fig. 3. We call the wedges adjacent to this ray left and right. The remaining wedge is upper. The rays are left, right, and downward. (Note that at most one of the wedges can be nonconvex.)

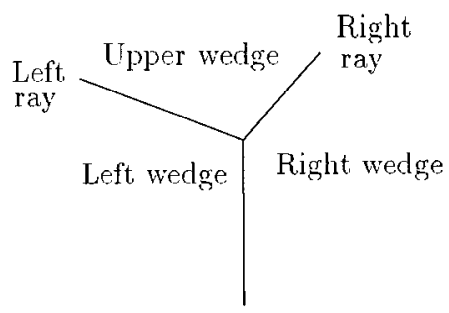

Downward ray

Fig. 3. The notation of a 3-cutting. 
Though our goal is to find 3-cutting whose rays do not pass through data points, it is convenient to define a canonical red 3-cutting for a triple $\left(g_{1}, g_{2}, g_{3}\right)$ that violates this condition. Let $p$ be a point in the plane and let $p_{1}, \ldots, p_{g n}$ be a list of red points in clockwise order starting from (but omitting) the downward ray from $p$. (If $p$ is red, then $p_{1}=p$. For a red point $q$ directly below $p, p_{g n}=q$.) The points $p_{g_{1} n}$ and $p_{\left(g_{1}+g_{2}\right) n}$ define a red 3-cutting with apex $p$. A canonical blue 3-cutting is defined similarly.

\section{A 3-Cutting Exists}

Note that any $\left(g_{1}, g_{2}\right)$-equitable 2-cutting can be viewed as a $\left(g_{1}, g_{2}, 0\right)$-equitable 3 cutting. Thus the Ham Sandwich Theorem implies that Theorem 2 is true for even $g$. The case of odd $g$ is more complicated.

\subsection{Odd $g \geq 3$}

We use the following topological lemma (a variant of one from [9]).

Lemma 4. Let $\mathcal{R}$ be any closed region in the plane. Let $A$ be a finite arrangement of curves in $\mathcal{R}$, in which each face of $A$ is labeled with a 1,2, 3, or 4 . Suppose that there are points $p$ and $q$, labeled 1 and 3 , on the boundary $\delta \mathcal{R}$ such that one component of $\delta \mathcal{R}-\{p, q\}$ uses labels 1, 2, 3 and the other component uses 1, 3, 4 (Fig. 4). Then some point of $\mathcal{R}$ lies on the boundary between two faces whose labels differ by two.

Proof. Subtract the faces labeled 2 and 4 from $\mathcal{R}$, and walk along the boundary of the resulting region starting from $p$. We either reach $q$, in which case we have seen a 1-3 transition, or we return to $p$, in which case we have walked from one component of $\delta \mathcal{R}-\{p, q\}$ to the other, and have seen a 2-4 transition.

The following theorem provides an equitable 3-cutting or 2-cutting for a given numbers of points in groups.

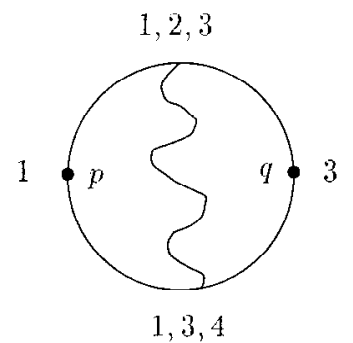

Fig. 4. 1-3 or 3-4 transition. 


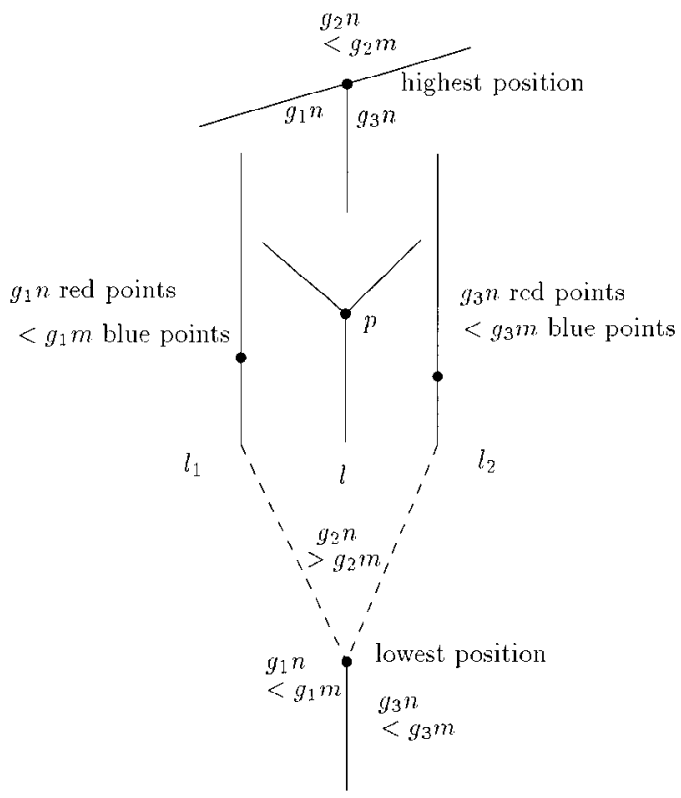

Fig. 5. The region $\mathcal{R}$ for triple $\left(g_{1}, g_{2}, g_{3}\right)$.

Theorem 5. Let $R$ be any set of $g n$ red points and let $B$ be any set $g m$ blue points in the plane in general position. Let $g_{1}, g_{2}$, and $g_{3}$ be positive integers with $g_{1}+g_{2}+g_{3}=g$. If $s\left(g_{1}\right)=s\left(g_{2}\right)=s\left(g_{3}\right)$, then there exists either

- $a\left(g_{1}, g_{2}, g_{3}\right)$-equitable 3-cutting, or

- $a\left(g_{i}, g-g_{i}\right)$-equitable 2-cutting for some $i$.

Proof. We can assume the sign $s\left(g_{1}\right)=-1$; if $s\left(g_{1}\right)=1$ we exchange the colors red and blue. We define a region $\mathcal{R}$ and label each $p \in \mathcal{R}$ as follows: Let $\mathcal{R}$ consist of those points in which the canonical red 3-cutting for triple $\left(g_{1}, g_{2}, g_{3}\right)$ with apex $p$ are convex (Fig. 5). Recall that no vertical line contains more than one data point. Let $x_{1}, \ldots, x_{g n}$ be a sorted list of $x$-coordinates of red points. Consider two vertical lines $l_{1}$ and $l_{2}$ passing through the $g_{1} n$th and $\left(g_{1}+g_{2}\right) n$th coordinates, i.e., $x=x_{g_{1} n}$ and $x=x_{\left(g_{1}+g_{2}\right) n}$. The closed halfplane left of $l_{1}$ contains exactly $g_{1} n$ red points and less than $g_{1} m$ blue points since $s\left(g_{1}\right)=-1$. Symmetrically, the open halfplane right of $l_{2}$ contains exactly $g_{3} n$ red points and less than $g_{3} m$ blue points since $s\left(g_{3}\right)=-1$. For any point left of $l_{1}$, the left wedge of a canonical red 3-cutting is nonconvex. Symmetrically, for any point right of $l_{2}$, the right wedge of a canonical red 3 -cutting is nonconvex. Hence the region $\mathcal{R}$ is bounded by the lines $l_{1}$ and $l_{2}$. Note that an open strip between the lines $l_{1}$ and $l_{2}$ contains exactly $g_{2} n$ red points and greater than $g_{2} m$ blue points.

Consider any vertical line $l$ between $l_{1}$ and $l_{2}$. For a point $p$ on $l$, the angles of left and right wedges of the canonical red 3-cutting are both less than $\pi$. Furthermore, each such angle is a monotone function of the $y$-coordinate of the point $p$ (the angles decrease when $p$ goes up). It follows that the angle of the upper wedge is monotone. If the point 

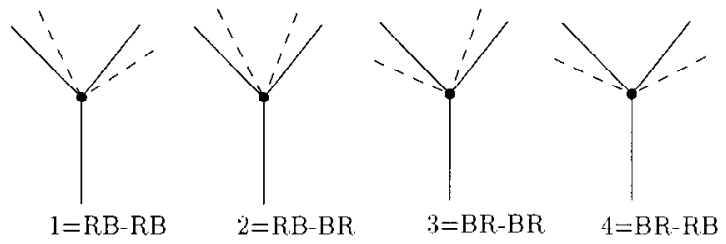

Fig. 6. The labels. The dashed rays are blue.

$p$ has the $y$-coordinate less than the minimum of $y$-coordinates of data points, then both the left and right rays go up and the upper wedge is convex. For a point $p$ on $l$ above all $y$-coordinates of red and blue points, the upper wedge of the canonical red 3-cutting has an angle greater than $\pi$. Hence there is a point on the line $l$ such that the points below $p$ belong to $\mathcal{R}$.

The lines passing through pairs of points of $R \cup B$ and the vertical lines through the points of $R \cup B$ form an arrangement $A$. For any point $p$ from the interior of a face $f \in A$, the left (right) rays of the two canonical cuttings are distinct. We are interested in the clockwise order of left rays (RB or BR) and the clockwise order of the right rays of the two canonical cuttings. We assign a label to $p$ according to $1=\mathrm{RB}-\mathrm{RB}, 2=\mathrm{RB}-\mathrm{BR}$, $3=\mathrm{BR}-\mathrm{BR}$, and $4=\mathrm{BR}-\mathrm{RB}$ (Fig. 6). All points of the same face of the arrangement $A$ have the same label, which is taken to be the label of the face.

Consider the labels on the boundary of $\mathcal{R}$. At $y=-\infty$ all labels are $2=\mathrm{RB}-\mathrm{BR}$ due to the signs of $g_{1}$ and $g_{3}$. The faces forming the left boundary of $\mathcal{R}$, all of which are incident on and right of the line $l_{1}$, have labels RBxx by the sign of $g_{1}$, which implies labels 1 or 2. Symmetrically, the faces of the right boundary of $\mathcal{R}$ have labels xxBR, which implies labels 2 or 3 . Along the remaining boundary of $R$, the red partition uses a straight line at the top, so the sign of $g_{2}$ rules out label $2=\mathrm{RB}-\mathrm{BR}$. The top face along the line $l_{1}$ has label $1=\mathrm{RB}-\mathrm{RB}$. The top face along the line $l_{2}$ has label $3=\mathrm{BR}-\mathrm{BR}$. By Lemma 4, therefore, we must have a 1-3 or 2-4 transition. This implies the existence of an equitable 3-cutting by Lemma 6.

Lemma 6. Let $p$ be a point of the region $\mathcal{R}$ that lies on the boundary between two faces whose labels differ by two. One of the faces around p contains the apex of an equitable 3-cutting.

Proof. First consider a special case where no data point lies below the apex $p$ (Fig. 7). Let $q$ be a point in a face whose boundary contains the point $p$. Let $r_{1}$ and $r_{2}$ be red points that define canonical red 3-cutting at $q$, with $\overrightarrow{q r}_{1}$ being the left ray. Similarly, let $b_{1}$ and $b_{2}$ be blue points that define canonical blue 3-cutting at $q$. Actually these points define canonical red and blue 3-cuttings for all points around $p$. If the line passing through the points $r_{1}$ and $b_{1}$ does not contain $p$, then the order of rays $\overrightarrow{q r}_{1}$ and $\overrightarrow{q b_{1}}$ is still the same when the point $q$ is rotated around $p$. This implies that the faces around $p$ use only two consecutive labels ( $1=\mathrm{RB}-\mathrm{RB}, 2=\mathrm{RB}-\mathrm{BR}$ or $3=\mathrm{BR}-\mathrm{BR}, 4=\mathrm{BR}-\mathrm{RB})$ contradicting the assumptions. Hence the points $r_{1}$ and $b_{1}$ lie on a ray emanating from $p$. Similarly, some ray emanating from $p$ intersects the points $r_{2}$ and $b_{2}$. The required 


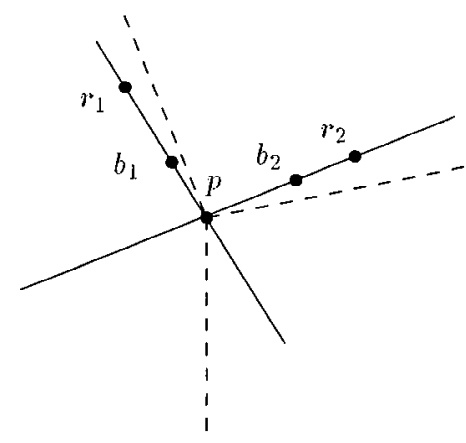

(a)

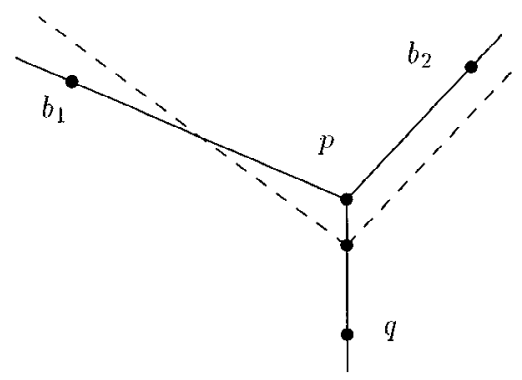

(b)

Fig. 7. Construction of an equitable 3-cutting (dashed rays) for a point $p$ with a 2-transition of labels. (a) No point below $p$. (b) The red point $q$ below $p$.

equitable 3 -cutting can be obtained by rotating the rays $\overrightarrow{p b_{1}}$ and $\overrightarrow{p b_{2}}$ by a sufficiently small angle clockwise.

Now suppose some point $q$, say red, lies below $p$ or coincides with $p$. Clearly, all canonical blue 3-cuttings for points in a sufficiently small ball with center at $p$ are defined by the same blue points, say $b_{1}$ and $b_{2}$. We prove that an equitable 3 -cutting $C$ can be obtained by a translation of the blue 3-cutting at $p$ downward by a sufficiently small distance and a rotation of the left ray by a sufficiently small angle clockwise such that the data points on the ray $\overrightarrow{p b_{1}}$ (except $p$, if $p \in R$ ) remain in the left wedge. The 3-cutting $C$ divides the blue points into subsets of $g_{1} m, g_{2} m$, and $g_{3} m$ points.

Split the ball containing $p$ by a vertical line passing through $p$. Let $H$ be the left open halfball. If the orders of left rays of red and blue 3-cuttings for some points in $H$ are changed (in other words there are two points in $H$ with labels RBxx and BRxx), then there is a red point $r_{1}$ in the ray $p b_{1}$ that forms all left rays of the red 3-cuttings for points in $H$. Therefore the left wedge of $C$ consist of $g_{1} n$ red points. Otherwise the change of the order of left rays for points whose labels differ by two is caused by inserting the red point $p$ into the left wedge of a red 3-cutting (with an apex in the right halfball). It follows that the left wedge of $C$ again consist of $g_{1} n$ red points.

Using a similar argument, we can show that the number of red points in the right wedge of $C$ is $g_{3} n$. Note that the right wedge of $C$ is convex because it is covered by a convex right wedge of red 3-cutting for a point with a label xxRB. Hence $C$ is an equitable 3 -cutting.

It remains to show how to construct an appropriate triple $\left(g_{1}, g_{2}, g_{3}\right)$.

\subsection{Finding $\left(g_{1}, g_{2}, g_{3}\right)$}

To apply Theorem 5 we need a triple $\left(g_{1}, g_{2}, g_{3}\right)$ with $g_{1}+g_{2}+g_{3}=g, 0 \leq g_{i}<g$, and signs $s\left(g_{1}\right)=s\left(g_{2}\right)=s\left(g_{3}\right)$. Recall that the signs are defined by vertical cuts. We 
can apply Lemma 3 if the $i$ th vertical line cuts off less (greater) than $i m$ blue points and the $(g-i)$ th vertical line cuts off greater (less) than $i m$ blue points. In other words the signs $s(i)$ and $s(g-i)$ are the same. To apply Theorem 5 we need a triple with the same signs. The following theorem provides one of these conditions.

Theorem 7. For any sequence of signs $s(1), \ldots, s(g-1)$, there is a pair $\left(g_{1}, g_{2}\right)$ or a triple $\left(g_{1}, g_{2}, g_{3}\right)$ with sum $g$ and the same signs.

Proof. If the pair $(1, g-1)$ has the same signs, we are done. Suppose $s(1) \neq s(g-1)$. Let $k$ be the smallest index with $s(k) \neq s(1)$ and $s(k-1)=s(1)$. Then either $s(k)=$ $s(g-k)$ or the triple $(1, k-1, g-k)$ has the same signs.

In fact one of the numbers in the triple can be specified.

Corollary 8. For any sequence of signs $s(1), \ldots, s(g-1)$, there is a pair $\left(g_{1}, g_{2}\right)$ or a triple $\left(g_{1}, g_{2}, 1\right)$ with sum $g$ and the same signs.

Applying Theorem 7 directly adds extra factor $g$ to the running time of constructing a subdivision of the plane into $g$ regions. The following theorem reduces this factor to $\log g$.

Theorem 9. For any sequence of signs $s(1), \ldots, s(g-1)$, there is a pair $\left(g_{1}, g_{3}\right)$ or a triple $\left(g_{1}, g_{2}, g_{3}\right)$ with sum $g$ and the same signs such that any $g_{i} \leq\lfloor 2 g / 3\rfloor$.

Proof. For even $g$ the pair $(g / 2, g / 2)$ has the same signs. Suppose that $g=2 k+1$. We can assume $s(1)=-1$; otherwise we exchange the colors red and blue. If $s(k)=-1$, then triple $(1, k, k)$ satisfies the theorem, so suppose $s(k)=1$. If $s(k+1)=1$, then the pair $(k, k+1)$ satisfies the theorem, so suppose $s(k+1)=-1$. Let $k_{1} \leq k$ be the number such that $s(j)=1$ for all $j, k_{1}<j \leq k$ and $s\left(k_{1}\right)=-1$. Denote $k_{2}=\lfloor g / 3\rfloor$.

Suppose $k_{1} \geq k_{2}$. Then the triple $\left(1, k_{1}, g-k_{1}-1\right)$ has the same signs since $s(g-$ $\left.k_{1}-1\right)=-s\left(k_{1}+1\right)=-1$. Note that $g-k_{1}-1 \leq g-\lfloor g / 3\rfloor-1$. One can prove $g-\lfloor g / 3\rfloor-1 \leq\lfloor 2 g / 3\rfloor$ by considering all residues of $g$ modulo 3 .

Alternatively $k_{1}<k_{2}$. In this case $g>5$ because $k_{2}=1$ for $g=5$. It follows that $g>6$ and $k_{2}+1=\lfloor g / 3\rfloor+1 \leq g / 3+1<(g+1) / 2=k$. Hence $s\left(k_{2}+1\right)=1$. The final triple with the same signs depends on the residue of $g$ modulo 3 :

- If $g=3 k_{2}$, then the triple $\left(k_{2}, k_{2}, k_{2}\right)$ has the same signs.

- If $g=3 k_{2}+1$, then the triple $\left(k_{2}, k_{2}, k_{2}+1\right)$ has the same signs.

- If $g=3 k_{2}+2$, then the triple $\left(k_{2}, k_{2}+1, k_{2}+1\right)$ has the same signs.

\section{Subdivision Theorems}

Applying Theorem 2 in a divide-and-conquer fashion we prove the main result of our paper. 
Theorem 10 (Equitable Subdivision). Given gn red and gm blue points in the plane in general position, there exists a subdivision of the plane into $g$ convex regions each of which contains $n$ red and $m$ blue points.

The 3-Cutting Theorem and the Equitable Subdivision Theorem are discrete. In the rest of this section we prove continuous versions of these theorems.

Let $\rho_{1}$ and $\rho_{2}$ be measurable functions $\mathrm{R}^{2} \rightarrow[0, \infty)$ with $\int_{\mathrm{R}^{2}} \rho_{i} d x=1$. A convex 3-cutting of the plane into wedges $W_{1}, W_{2}$, and $W_{3}$ is equitable (or more specifically $\left(g_{1}, g_{2}, g_{3}\right)$-equitable) if $\int_{W_{i}} \rho_{1} d x=\int_{W_{i}} \rho_{2} d x=g_{i} / g$ for some integers $0 \leq g_{1}, g_{2}, g_{3}<g$ with $g_{1}+g_{2}+g_{3}=g$.

Theorem 11 (3-Cutting, Continuous Version). Let $\rho_{1}$ and $\rho_{2}$ be measurable functions $\mathrm{R}^{2} \rightarrow[0, \infty)$ with $\int_{\mathrm{R}^{2}} \rho_{i} d x=1$. For any integer $g \geq 2$, there exists an equitable 3-cutting.

Proof. Note that, for $g=2$, this is the continuous Ham Sandwich Theorem. Fix any integer $g \geq 3$. The idea is to approximate functions $\rho_{i}$ using a continuous version of the Ham Sandwich Theorem. Consider the function $\rho_{1}$. For a region $R$, the weight of $R$ is $\int_{R} \rho_{1}(x) d x$. The weight of the plane is 1 . Draw any line $l_{1}$ (for example, vertical) that defines halfplanes $H_{1}$ and $H_{2}$ of weight $\frac{1}{2}$. Define functions $\varphi_{1}$ and $\varphi_{2}$ on the plane by

$$
\varphi_{i}(p)= \begin{cases}2 \rho_{1}(p) & \text { if } p \in H_{i} \\ 0 & \text { otherwise }\end{cases}
$$

Applying the continuous version of the Ham Sandwich Theorem we construct a line $l_{2}$ that halves both $H_{1}$ and $H_{2}$, producing four wedges of weight $\frac{1}{4}$.

We partition each of the four wedges into four regions in the same fashion. The number of convex regions after partitioning $k$ times is $4^{k}$. This subdivision $S$ satisfies a line property that any line properly intersects at most $3^{k}$ regions. The line property can be proved using the fact that a line cannot contain four interior points of wedges formed by two lines.

Pick a red point in each region. We define $4^{k}$ blue points in similar way using the function $\rho_{2}$. To apply Theorem 2 the number of red and blue points must be an integer multiple of $g$. We remove any $r=4^{k}-g\left\lfloor 4^{k} / g\right\rfloor$ red and blue points. By 3-cutting Theorem 2, for any $k$, there exists an equitable 3-cutting of $g\left\lfloor 4^{k} / g\right\rfloor$ red and blue points. Let it be a $\left(g_{1}, g_{2}, g_{3}\right)$-equitable 3 -cutting of the plane into the wedges $W_{1}, W_{2}$, and $W_{3}$. By the line property, each ray of the 3 -cutting crosses at most $3^{k}$ convex regions of $S$. The wedge $W_{i}$ contains at least $g_{i}\left\lfloor 4^{k} / g\right\rfloor-2 \cdot 3^{k}-r$ whole regions of $S$ and intersects at most $g_{i}\left\lfloor 4^{k} / g\right\rfloor+2 \cdot 3^{k}+r$ regions of $S$. Each region of $S$ has weight $1 / 4^{k}$. Hence

$$
g_{i}\left\lfloor\frac{4^{k}}{g}\right\rfloor-2 \cdot 3^{k}-r \leq 4^{k} \int_{W_{i}} \rho_{1} d x \leq g_{i}\left\lfloor\frac{4^{k}}{g}\right\rfloor+2 \cdot 3^{k}+r
$$

or

$$
\left|4^{k} \int_{W_{i}} \rho_{1} d x-g_{i}\left\lfloor\frac{4^{k}}{g}\right\rfloor\right| \leq 2 \cdot 3^{k}+r
$$


Using $r=4^{k}-g\left\lfloor 4^{k} / g\right\rfloor$ we obtain

$$
\left|4^{k}\left(\int_{W_{i}} \rho_{1} d x-\frac{g_{i}}{g}\right)+r \frac{g_{i}}{g}\right| \leq 2 \cdot 3^{k}+r
$$

or

$$
4^{k}\left|\int_{W_{i}} \rho_{1} d x-\frac{g_{i}}{g}\right| \leq 2 \cdot 3^{k}+r+r \frac{g_{i}}{g} \leq 2 \cdot 3^{k}+2 r .
$$

Hence

$$
\left|\int_{W_{i}} \rho_{1} d x-\frac{g_{i}}{g}\right| \leq 2\left(\frac{3}{4}\right)^{k}+2 \frac{g}{4^{k}} .
$$

One can prove the same bound for $\rho_{2}$ :

$$
\left|\int_{W_{i}} \rho_{2} d x-\frac{g_{i}}{g}\right| \leq 2\left(\frac{3}{4}\right)^{k}+2 \frac{g}{4^{k}} .
$$

The triples $\left(g_{1}, g_{2}, g_{3}\right)$ can be different if we change $k$. We extract an infinite sequence of numbers $k_{1}, \ldots, k_{i}, \ldots$ that define the same triples $\left(g_{1}, g_{2}, g_{3}\right)$. Let $p_{i}$ be an apex of the 3-cutting constructed for $k=k_{i}$. It is clear that $p_{i}$ lies in the vertical slab defined by the lines $x=a$ and $x=b$ such that

$$
\int_{x<a} \rho_{1} d x=\int_{x>b} \rho_{1} d x=\frac{1}{g} .
$$

We can extract an infinite subsequence of apexes $p_{i_{i}}, p_{i_{2}}, \ldots$ that converges to some point $p \in \mathrm{R}^{2}$ or to an infinite point $(x, \pm \infty)$. In the first case the wedges of corresponding 3 -cuttings converge to some wedges $W_{1}, W_{2}$, and $W_{3}$ that define a $\left(g_{1}, g_{2}, g_{3}\right)$-equitable 3 -cutting. In the remaining case some two rays of 3-cuttings converge to vertical lines that define three slabs of weights $g_{1}, g_{2}$, and $g_{3}$ (for both functions $\rho_{1}$ and $\rho_{2}$ ). Each of these lines defines a 2-cutting that is a special case of 3-cutting.

Applying Theorem 11 recursively for regions with a number of groups more than one we can prove the following theorem.

Theorem 12 (Equitable Subdivision, Continuous Version). Let $\rho_{1}$ and $\rho_{2}$ be measurable functions $\mathrm{R}^{2} \rightarrow[0, \infty)$ with $\int_{\mathrm{R}^{2}} \rho_{i} d x=1$. For any integer $g>0$, there exists a subdivision of the plane into $g$ convex regions $R_{1}, \ldots, R_{g}$ such that

$$
\int_{R_{j}} \rho_{i} d x=\frac{1}{g}, \quad \text { for } i=1,2 \text { and } j=1, \ldots, g .
$$

\section{Algorithm}

We next describe an algorithm that finds discrete 3-cuttings recursively. The recursive procedure has input $g, n, m$ and two sets $R$ and $B$ of points. If $g$ is even we use the linear time algorithm of Lo et al. for the Ham Sandwich Problem [13]. Otherwise provided $g>1$ we find a triple $\left(g_{1}, g_{2}, g_{3}\right)$ using Theorem 9, and find an equitable 3-cutting by Theorem 5 (in fact, the algorithm may find 2-cutting instead of 3-cutting). For each wedge with $g_{i} n$ red and $g_{i} m$ blue points, we continue recursively. 


\subsection{Computing a Triple $\left(g_{1}, g_{2}, g_{3}\right)$}

We need a triple of integers $0 \leq g_{1}, g_{2}, g_{3} \leq g$ that sum to $g$ and have the same signs $s\left(g_{1}\right)=s\left(g_{2}\right)=s\left(g_{3}\right)$. To compute the signs $s(1), \ldots, s(g-1)$ we construct $g-1$ vertical lines separating red points into $g$ sets of equal size. This can be done in linear time if the red points are presorted. Presorting takes $O(N \log N)$ time. We can count the number of blue points in the $g$ strips in linear time if the blue points are presorted. For each vertical line, we compute the number of blue points to the left of the line. Comparing $i$ th number with $i m$ gives sign $s(i)$. If, for some $i$, the $i$ th number coincides with $i m$, then the $i$ th vertical line gives the equitable 2-cutting.

Now we can find the triple $\left(g_{1}, g_{2}, g_{3}\right)$ using Theorem 9 .

\subsection{One More Topological Lemma}

The most difficult part of the algorithm is finding an equitable 3-cutting. Recall that our proof that an equitable 3-cutting exists depends on the fact (Lemma 4) that in a certain arrangement with faces labeled 1,2,3, or 4, there are adjacent faces whose labels differ by two. Unfortunately, Lemma 4 does not give a way to compute two such faces except to look through all faces. We give a general topological lemma for the existence of such faces that supports binary search.

Let $G$ be a dual graph of the arrangement $A$ formed by the lines passing through pairs of points of $R \cup B$ and the vertical lines through the points of $R \cup B$. The vertices of $G$ correspond to the faces and two vertices are adjacent iff corresponding faces are adjacent. The graph $G$ is planar. We associate the points $(1,0),(0,1),(-1,0)$, and $(0,-1)$ with the labels 1,2, 3, and 4, respectively. Let $S$ be the square with these vertices. We label the vertices of the graph $G$ by the points associated with labels of corresponding faces. Note that the labels of two vertices corresponding to faces whose labels differ by two form a diagonal of the square $S$. If two such vertices are adjacent, we call an edge connecting them a diagonal.

If the exterior face of the graph $G$ contains a diagonal edge we are done. Consider a nondiagonal directed edge $e=(u, v)$ of $G$. It corresponds to a directed side of the square $S$. We assign a directed length to the edge $e$ to be 1 (resp. -1 ) if the corresponding directed side of the square $S$ has clockwise (resp. counterclockwise) direction. A directed length of a path $P$ without diagonal edges is the sum of directed lengths of edges of $P$.

Recall the labels of the boundary of the region $\mathcal{R}$. The top has labels 1,2 , or 4 , the bottom has 2 , the left side has 1,2 , and the right side has 2,3 . This means that any closed path along the boundary of $G$ clockwise has the directed length 4 .

Observation 13. The length of any closed path without diagonal edges is an integer multiple of four.

Proof. The length of a path $P$ does not change if we remove two consecutive edges with opposite directed lengths. After all deletions the path $P$ consists of edges with the same directed length 1 or -1 . The length of a path $P$ modulo 4 does not change if we remove any four consecutive edges. Now the path $P$ contains only one point. 
We define a number of turns of a closed path $P$ to be the directed length of $P$ divided by four. So the clockwise boundary of $G$ has one turn. Now we can formulate the topological lemma.

Lemma 14. Let $G$ be a connected graph embedded to the plane and the vertices of $G$ are labeled by the vertices of the square $S$. If the boundary of $G$ has an odd number of turns, then there are two vertices of the same face of $G$ whose labels form a diagonal of $S$.

Proof. The proof is constructive and can be used in a binary search. If the graph $G$ is not a cycle, we can find a cutting path $P$ with only two vertices in the boundary of $G$ that are ends of $P$. The path $P$ divides $G$ into two subgraphs $G_{1}$ and $G_{2}$ (both graphs contain $P$ ). The clockwise directed length of $G$ is equal to the sum of clockwise directed lengths of subgraphs because each edge of $P$ is included twice in the sum with opposite signs. It follows that the number of turns of the boundary of $G$ is the sum of the number of turns of the boundaries of the subgraphs. One of the subgraphs has an odd number of turns because its sum is odd, so we have reduced the graph.

If the graph $G$ cannot be reduced, then $G$ is a cycle with one bounded face. The vertices of $G$ use all labels (otherwise the directed length of a cycle is zero). Hence there are two vertices that form a diagonal.

\subsection{Binary Search}

Given a triple $\left(g_{1}, g_{2}, g_{3}\right)$ from Theorem 9. The algorithm finds an equitable 3-cutting. First we determine the location of an apex of 3-cutting among the vertical lines passing through red and blue points. We apply the binary search on $x$-coordinates of data points. Actually, each data point $p$ gives two vertical cuts of the region $\mathcal{R}$, left and right of $p$, say $x=p_{x}+\varepsilon$ and $x=p_{x}-\varepsilon$ for a sufficiently small $\varepsilon$ (blue point from the line $l_{i}, i=1,2$, gives only one cut).

The input of the binary search procedure is a strip between two vertical lines, a cutting line $l$, two directed lengths $d_{1}$ and $d_{2}$ of the left and right boundary of the region in the strip in clockwise order and labels $t_{1}$ and $t_{2}$ of the top faces. The boundary of a graph corresponding to the region has an odd numbers of turns. The algorithm computes a top label $t$ and a directed length $d$ of $l$ (in the down-up direction) induced by the faces. The cutting line $l$ makes two subgraphs whose boundary lengths can be computed using lengths $d_{1}, d_{2}$, and $d$ and top labels $t_{1}, t_{2}$, and $t$ (note that all top paths do not use label 2). One of the subgraphs has an odd number of turns. The binary search proceeds to this subgraph.

The main problem is to compute the length $d$. A vertical cut of the region $\mathcal{R}$ intersects $\Omega\left(N^{2}\right)$ faces of the arrangement $A$. Fortunately, the faces form sequences with the same labels and the number of such sequences is at most $O\left(N^{4 / 3}\right)$. Consider a set of red points to the left of the cutting line $l$. The left ray of any red 3-cutting with an apex on the line $l$ cuts off a fixed number $g_{1} n$ of red points.

In this way the problem of computing the length of a cutting line is reduced to a well-studied $k$-level problem in a dual setting, see [1] for a recent survey. Suppose we 


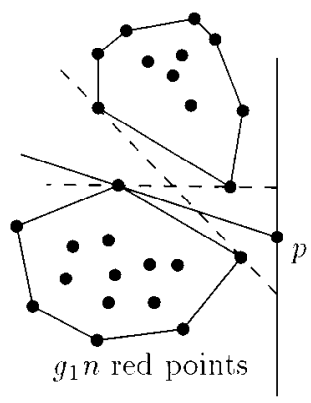

Fig. 8. Convex hulls.

are given an arrangement of $N$ lines in the dual plane in general position. A point $p$ from on one of the lines of the arrangement has level $k$ if exactly $k$ other lines pass below $p$. The $k$-level is the union of all such points of level $k$, a piecewise-linear curve. Recall that the $k$-level problem is to find $\psi_{k}(N)$, the maximum possible number of segments that can be on the $k$-level of an arrangement of $N$ lines. Let $\psi(N)$ be the maximum of the $\psi_{k}(N)$ problem over all $k$. Dey [4] recently showed that $\psi_{k}(N)=O\left(N k^{1 / 3}\right)$, so $\psi(N)=O\left(N^{4 / 3}\right)$. The $k$-level reporting problem is to describe the vertices that form the $k$-level, in order of $x$-coordinate.

The points of the $\left(g_{1} n-1\right)$-level correspond to the lines cutting off $g_{1} n$ red points (Fig. 8). Each segment of the $\left(g_{1} n-1\right)$-level corresponds to the set of lines that cut off $g_{1} n$ red points and pass through the same red point. In this way the $\left(g_{1} n-1\right)$-level induces a partition of $l$ into segments. The number of such segments is $O(\psi(N))$. The blue points also generate a partition of the line $l$ into $O(\psi(N))$ segments. (If the halfplane left of $l$ contains less than $g_{1} m$ blue points, all left wedges of blue canonical 3-cuttings are nonconvex and the order of left rays is RB.) Combining both partitions we divide the line $l$ into $O(\psi(N))$ segments such that, for all apexes from the same segment, the left rays of red and blue 3 -cuttings pass through the same pair of red and blue points $p_{\mathrm{r}}$ and $p_{\mathrm{b}}$. Hence the order of the left rays can change at most one time along a segment, if it is crossed by the line passing through $p_{\mathrm{r}}$ and $p_{\mathrm{b}}$. Symmetrically, the points to the right of $l$ generate $O(\psi(N))$ segments with an unchangeable order of right rays. Combining all segments we obtain $O(\psi(N))$ segments such that the points from the same segment have the same labels.

The algorithm uses four current segments in one of four combining sets. For a segment, we maintain two convex hulls of subsets that are divided by a corresponding ray. We use the dynamic algorithm by Overmars and van Leeuwen [15] that maintains a convex hull in $O\left(\log ^{2} N\right)$ time per update (see [8], for example). Thus we obtain an $O\left(\psi(N) \log ^{2} N\right)$ algorithm to compute a directed length $d$. Therefore we proved the following theorem.

Theorem 15. Let $T(N)$ be the running time of an algorithm for reporting a k-level. The binary search can be implemented in $O(T(N) \log N)$ time.

Corollary 16. The binary search can be implemented in $O\left(N^{4 / 3} \log ^{3} N\right)$ using the algorithm of Overmars and van Leeuwen [15]. 


\subsection{Searching in the Strip}

The binary search can be interrupted if one of the vertical cuts contains a diagonal. We find an equitable 3-cutting in linear time using Lemma 6. Otherwise the binary search outputs a vertical strip $s$ with at most one data point in the interior of $s$. If $s$ contains one data point, then there are two neighbor segments whose labels differ by two. We again apply the construction of Lemma 6.

Consider the case of the empty strip $s$. Of course our technique would allow us to continue the binary search among all crossing points of the arrangement $A$. However, locating the appropriate vertical cuts seems difficult. Actually the final strip $s$ defines the set of left and right rays and we can find two rays with a crossing point inside $s$. The strip $s$ contains an apex of the solution but no data points. We look through the sides of the strip but we change their roles. We use the partition of the line $l_{1}$ generated by the points to the right of the strip. Similarly the line $l_{2}$ is partitioned by the points to the left of the strip. The difference is that we use two pointers for each color. For the line $l_{2}$, one colored pointer indicates the $g_{1} n$th point of the same color, the second pointer indicates the $\left(g_{1} n+1\right)$ th point. So, for a point of the line $l_{2}$, we know the range of left red rays. The total number of the segments into which the line $l_{2}$ is divided is still $O(\psi(N))$. We also define the blue range and two ranges for the line $l_{1}$. Note that the ranges of each segment are defined by four points (two red and two blue). We exclude the intersection of the lines passing through these points and the segment by dividing the segment.

Now we are ready to start a climb. We pass segments from a side of the strip if the red and blue ranges do not intersect. Otherwise all rays emanating from a point of a segment lie between the rays emanating from the endpoints of the segment and form a trapezoid in the strip $s$, for example, the trapezoid $A B C D$ in Fig. 9. Suppose both segments from $l_{1}$ and $l_{2}$ form trapezoids. If two trapezoids intersect, then any point of the intersection gives an equitable 3-cutting, as in the dashed lines of Fig. 9. Otherwise one of the trapezoids lies below the other. The lower trapezoid does not contain an apex of

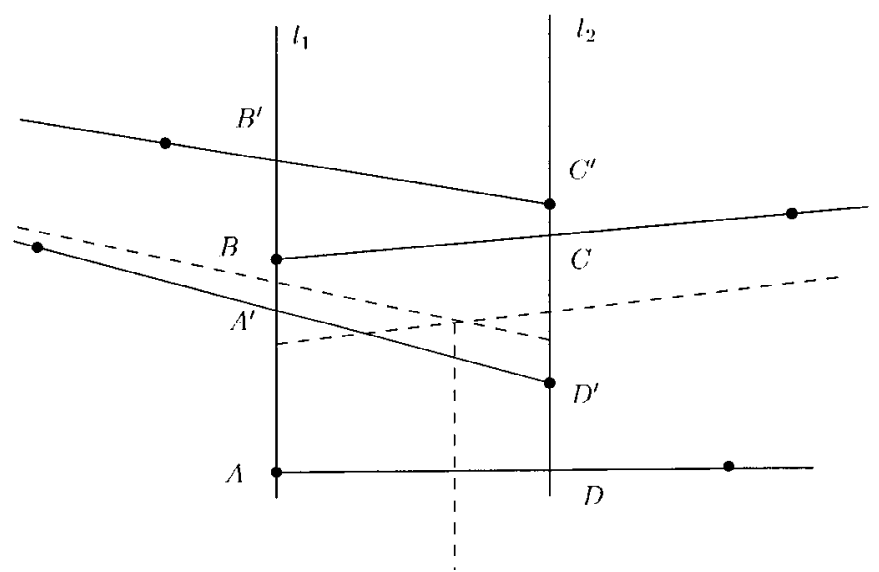

Fig. 9. Searching in the strip. Two extreme rays $A D$ and $B C$ form a trapezoid $A B C D$. Two intersecting trapezoids $A B C D$ and $A^{\prime} B^{\prime} C^{\prime} D^{\prime}$ give an equitable 3-cutting (dashed rays). 
an equitable 3-cutting by invariant. We pass the segment corresponding to this trapezoid. Climbing will eventually stop because the strip contains a solution.

\subsection{Running Time Analysis}

The running time for the searching in the strip is $O\left(N^{4 / 3} \log ^{2} N\right)$. The total running time of the 3-cutting algorithm is $O\left(N^{4 / 3} \log ^{3} N\right)$. To obtain a planar subdivision we use Theorem 9, which gives extra factor $\log g$. Therefore we proved the following theorem.

Theorem 17. Given gn red points and gm blue points in the plane in general position. A subdivision of the plane into $g$ convex polygonal regions each of which contains $n$ red and $m$ blue points can be computed in $O\left(N^{4 / 3} \log ^{3} N \log g\right)$ time where $N=g(n+m)$.

\section{Discussion}

Recall that the conjecture of Kaneko and Kano asserts the existence of disjoint convex polygons. Such polygons do not guarantee a subdivision of the plane into convex polygonal regions. Figure 10(a) shows an example of such polygons. There is no convex subdivision of the plane into three polygonal regions such that each region contains one of these polygons. Actually our 3-cutting theorem induces a more restricted class of subdivisions. Figure 10(b) gives an example of a subdivision of the plane that cannot be constructed by 3-cuttings.

\section{Conclusion}

We proved the existence of an equitable subdivision of the plane (Theorem 10). The special case of $n=m=1$ corresponds to the classical result that, for $g$ red points and $g$ blue points in general position, there is a perfect matching, i.e., $g$ disjoint segments connecting red and blue points.

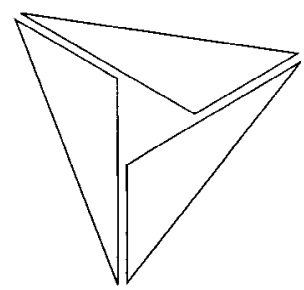

(a)

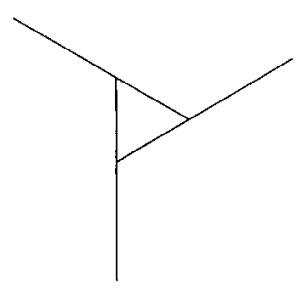

(b)

Fig. 10. (a) Three polygons that cannot be extended to a subdivision of the plane into three convex regions. (b) Polygonal subdivision that cannot be obtained by 3 -cuttings. 
The presented method can be used to derive the following subdivision result for an arbitrary number of red and blue points in an arbitrary position.

Theorem 18. Let $R$ and $B$ be finite sets of red and blue points in the plane. For any $g>0$, there exists a subdivision of the plane into $g$ convex polygonal regions so that the interior of each region contains at most $\lfloor|R| / g\rfloor$ red points and at most $\lfloor|B| / g\rfloor$ blue points.

Actually the proof of Theorem 5 allows us to restrict the 3-cuttings to be found. If $g n$ red and $g m$ blue points in general position do not admit 2-cutting, then there exists an equitable 3-cutting with a prescribed direction of one of the rays and the order of groups around apex. It is interesting that the sequence of signs $s(1), \ldots, s(g-1)$ is the source of various triples for the equitable 3-cuttings, for example, Theorems 7 and 9.

Finally it should be mentioned that our proof of the continuous version of 3-cutting (Theorem 11) can be used to produce an approximate algorithm for finding an equitable subdivision of two mass distributions in the plane using the 3-cutting algorithm (discrete version) and an approximate algorithm for 2-cutting.

\section{Acknowledgments}

We appreciate the helpful comments of I. Bárány, H. Edelsbrunner, and M. Kano.

\section{References}

1. P. Agarwal and M. Sharir. Arrangements. In Handbook of Computational Geometry (J.-R. Sack and J. Urrutia, eds.). Elsevier Science/North-Holland, Amsterdam, 1998.

2. I. Bárány. Geometric and combinatorial applications of Borsuk's theorem. In New Trends in Discrete and Computational Geometry (J. Pach, ed.). Vol. 10 of Algorithms and Combinatorics, pp. 235-249. SpringerVerlag, Berlin, 1993.

3. I. Bárány and J. Matoušek. Simultaneous partitions of measures by k-fans. Preprint, 1999.

4. T. K. Dey. Improved bounds on planar $k$-sets and related problems. Discrete Comput. Geom., 19 (1998), 373-382.

5. M. Díaz and J. O’Rourke. Ham-sandwich sectioning of polygons. In Proc. 2nd Canad. Conf. Comput. Geom., pp. 282-286, 1990.

6. D. P. Dobkin and H. Edelsbrunner. Ham-sandwich theorems applied to intersection problems. In Proc. 10th Internat. Workshop Graph-Theoret. Concepts Comput. Sci., pp. 88-99, 1984.

7. H. Edelsbrunner and R. Waupotitsch. Computing a ham-sandwich cut in two dimensions. J. Symbolic Comput., 2 (1986), 171-178.

8. H. Edelsbrunner and E. Welzl. Constructing belts in two-dimensional arrangements with applications. SIAM J. Comput., 15 (1986), 271-284.

9. J. E. Goodman, J. Pach, and C. K. Yap. Mountain climbing, ladder moving, and the ring width of a polygon. Amer. Math. Monthly, 96 (1989), 494-510.

10. H. Ito, H. Uehara, and M. Yokoyama. Two-dimension ham sandwich theorem for partitioning into three convex pieces. In Proc. Japan Conf. Discrete Comput. Geom., pp. 69-73, 1998.

11. A. Kaneko and M. Kano. Balanced partitions of two sets of points in the plane. In Proc. 10th Canad. Conf. Comput. Geom., 1998. Extended Abstract.

12. C.-Y. Lo, J. Matoušek, and W. Steiger. Ham-sandwich cuts in $\Re^{d}$. In Proc. 24th Annu. ACM Sympos. Theory Comput., pp. 539-545, 1992. 
13. C.-Y. Lo, J. Matoušek, and W. L. Steiger. Algorithms for ham-sandwich cuts. Discrete Comput. Geom., 11 (1994), 433-452.

14. C.-Y. Lo and W. Steiger. An optimal-time algorithm for ham-sandwich cuts in the plane. In Proc. 2nd Canad. Conf. Comput. Geom., pp. 5-9, 1990.

15. M. H. Overmars and J. van Leeuwen. Maintenance of configurations in the plane. J. Comput. System Sci., 23 (1981), 166-204.

16. T. Sakai. Radial partitions of point sets in $R^{2}$. In Proc. Japan Conf. Discrete Comput. Geom., pp. 74-78, 1998.

17. W. Steiger. Algorithms for ham sandwich cuts. In Proc. 5th Canad. Conf. Comput. Geom., p. 48, 1993.

18. R. T. Živaljević. Topological methods. In Handbook of Discrete and Computational Geometry (J. E. Goodman and J. O’Rourke, eds.), pp. 209-224. CRC Press, Boca Raton, FL, 1997.

19. R. T. Živaljević and S. T. Vrećica. An extension of the ham sandwich theorem. Bull. London Math. Soc., 22 (1990), 183-186.

Received February 19, 1999, and in revised form June 3, 1999. Online publication August 21, 2000. 